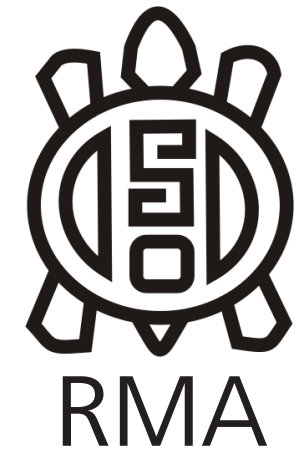

Arqueología

\title{
Tecnología lítica en la sierra de El Alto- Ancasti (Catamarca). Aporte desde la experimentación
}

\author{
Lithic technology at El Alto-Ancasti mountains (Catamarca). \\ Contributions from experimental analysis
}

Débora Egea

Centro de Investigaciones y Transferencia de Catamarca (CITCA), CONICET. Catamarca, Argentina. E-mail: deb.egea@gmail.com

\begin{abstract}
Resumen
El cuarzo es la materia prima dominante utilizada para la talla en las ocupaciones prehispánicas de la sierra de El Alto-Ancasti, superando el $95 \%$ en todas las muestras hasta el momento estudiadas. Un análisis experimental permitió profundizar el conocimiento sobre los modos de producción de los artefactos líticos en esta roca, haciendo hincapié en las distintas técnicas de talla identificadas, la talla directa y la talla bipolar. Estas actividades fueron desarrolladas con la finalidad de obtener muestras comparativas a los conjuntos arqueológicos recuperados en algunos de los sitios de la sierra y explorar el potencial de esta materia prima para la confección de instrumentos, en función de sus propiedades mecánicas y aptitudes para la talla. El estudio que se propone contribuirá a la interpretación de las prácticas y elecciones tecnológicas de las poblaciones serranas y su relación con los recursos locales. Se pretende aportar así al conocimiento sobre las ocupaciones humanas del área y brindar datos específicos a la temática a nivel regional, sumando nueva información acerca de la materialidad lítica en contextos agro-pastoriles, atendiendo especialmente al tratamiento de una materia prima relativamente poco estudiada como el cuarzo.
\end{abstract}

Palabras Clave: tecnología lítica; materia prima; cuarzo; arqueología experimental; sierra de El Alto-Ancasti.

\begin{abstract}
Quartz is the dominant raw material used for the manufacture of lithic technology in the pre-Hispanic occupations at the El Alto-Ancasti mountains, overcoming $95 \%$ in all the samples studied until now. An experimental analysis allows us to deepen into the knowledge on the quartz lithic artifacts production, emphasizing in the different techniques identified, the direct flaking and the bipolar flaking. The study was developed with the goal to obtain comparative samples to the archaeological sets recovered in some of the sites of the studied area and to explore the potential of this raw material for the manufacture of instruments, considering its mechanical properties. This article will contribute to the interpretation of the practices and technological choices of the prehispanic populations and its relation with the local resources. The aim is to contribute to the knowledge on the human occupations of the area and provide specific data on the subject at a regional level, adding new information about the lithic materiality in agro-pastoral contexts, and paying particular attention to the treatment of a relatively little studied raw material as the quartz.
\end{abstract}

Keywords: lithic technology; raw material; quartz; experimental archaeology; El Alto-Ancasti mountains.

Investigaciones realizadas en los últimos años en la sierra de El Alto-Ancasti (Catamarca, Argentina) han abordado temáticas referidas a las ocupaciones humanas durante el primer milenio de nuestra era (Dlugosz y Nuñez Regueiro, 2005; Quesada, Gastaldi, y Granizo, 2012; Nazar, Gheco, y De la Fuente, 2013; Gordillo, Zucarelli, y Eguia, 2017), aportando una visión acerca de la sierra, caracterizada por la presencia de ocupaciones campesinas, muy vinculadas con el ambiente local, con una fuerte inversión de trabajo tanto en la arquitectura doméstica como productiva y con intenso aprovechamiento de los recursos locales
(Quesada, et al., 2012; Moreno y Sentinelli, 2014; Moreno, 2015; Moreno y Egea, 2015; Ahumada y Moreno, 2015-2016; Egea, 2016). Tales evidencias, permiten asumir una forma de construcción del espacio social marcada por una gran cantidad de sitios de vivienda y espacios agrícolas elaborados, preparados para perdurar en el tiempo, lo que demuestra un compromiso a largo plazo con el territorio. A su vez, en los numerosos sitios con arte rupestre de la zona, se pudieron identificar diferencias dentro de lo que se consideraba, de manera uniforme, como arte rupestre de la cultura de La Aguada 
(González, 1998; Llamazares, 1999-2000; Gheco, 2017).

En este contexto, el estudio de la tecnología lítica aportó hasta el momento algunas líneas de evidencia relevantes, como la importancia de los recursos locales, materializado en el uso casi exclusivo del cuarzo y ciertas características generales de los artefactos utilizados y su historia de producción (Moreno y Sentinelli, 2014; Moreno, 2015; Moreno y Egea, 2015; Egea, 2016). Sin embargo, es necesaria y relevante la profundización de estos datos. Así, en este aporte nos focalizamos en el análisis experimental en cuarzo que permitirá entender los modos particulares de producción de los artefactos líticos de esta roca. El análisis tiene como fin indagar en las aptitudes y propiedades de esta materia prima para la confección de instrumentos.

El estudio que se propone contribuirá a la interpretación de las prácticas y elecciones tecnológicas de las poblaciones serranas y su relación con recursos locales, aportando al conocimiento sobre las ocupaciones humanas presentes en el área y sumando nueva información acerca de la materialidad lítica en contextos agro-pastoriles, atendiendo especialmente al tratamiento de una materia prima relativamente poco estudiada como el cuarzo.

Es importante destacar que la problemática de la tecnología lítica en contextos agro-pastoriles, a pesar de haber sido relativamente dejada de lado en la historia de las investigaciones, ha tenido un fuerte desarrollo en los últimos años, sobre todo en el paisaje puneño (Escola, 2004; Chaparro, 2005; Elías, 2012). Por su parte, en el caso de la tecnología lítica en contextos ambientales relativamente semejantes a los estudiados aquí, contamos con los avances realizados en distintas localidades de las Sierras Centrales (Pautassi y Sario, 2014; Sario y Pautassi, 2015; Robledo, Cattaneo, y Conte, 2017), los cuales abordan también la producción lítica en cuarzo.

\section{El cuarzo como materia prima para la confección de instrumentos}

El cuarzo es un mineral cristalino muy común y se da en varios tipos y colores. Tiene una dureza de siete en la escala de Mohs.

Se caracteriza por su abundante localización en el paisaje de la zona, su accesibilidad no presenta restricciones. Si bien esta materia prima aparece con frecuencia en los registros líticos del NOA, los estudios específicos sobre su empleo son relativamente escasos (Gaál, 2011; Moreno, 2015; Sario y Pautassi, 2015).

Esto puede estar relacionado con que las producciones líticas realizadas sobre cuarzo presentan serias dificultades de identificación de los rasgos diagnósticos, debido, en parte, al desconocimiento de las mecánicas de fractura de este tipo de material y su empleo en procesos de talla. En muchas publicaciones solo se menciona la presencia de esta materia prima caracterizándose por la falta de detalle y precisión al respecto, conduciendo a una falta de conocimiento de este tipo de rocas (Prous, 2004; Ballin, 2008; Frabegas Valcarse y Rodríguez Rellan, 2008).

Esto se debe, principalmente, a la alta dureza del cuarzo y su baja calidad para la talla, ya que puede presentar una estructura interna con abundancia de fisuras, diaclasas y otros defectos que hacen difícil controlar la fractura (Mourre, 1996; Spott, 2005; Pautassi y Sario, 2014; Moreno, 2015; Rodriguez Rellan, 2016). Debido a esto no se puede definir un comportamiento mecánico único para este mineral. Las distintas variedades van a reaccionar de manera diferente, dependiendo de su granulometría o de la existencia de planos de debilidad internos y el resultado de un mismo gesto técnico puede variar totalmente. Como resultado, sus cualidades técnicas difieren mucho de las descritas para materiales más comunes en la literatura arqueológica como la sílice. Estas características del cuarzo, a su vez obligan a considerar en los análisis el importante número de desechos que produce, eventualmente utilizables (Prous, 2004).

Positivamente, en el último tiempo empezaron a surgir trabajos que tienen en cuenta las características específicas de esta materia prima (Baqueiro Vidal, 2006; Fábregas Valcarce y Rodríguez Rellán, 2008; Driscoll, 2010; Sanches Yustos, Diez Martín, Domínguez-Rodrigo, y Tarriño Vinagre, 2012; Moreno y Sentinelli, 2014; Kutsson, Knutsson, Taipale, Tallavaara, y, Darmaek 2015; Pautassi, 2015; Caminoa, 2016; Rodriguez Rellan, 2016; Carrera, 2017; Reinoso, 2017). En los últimos años se ha producido una considerable innovación en los métodos de análisis de uso y desgaste para este tipo de materias primas, junto con el desarrollo de varias técnicas analíticas originales para evaluar entre diferentes patrones de desgaste (Cousseran, 2002; Ballin, 2008; Lombera Hermida, 2008; Manninen, 2016).

\section{Área de estudio}

La sierra de El Alto-Ancasti se ubica en el este de la provincia de Catamarca (Figura 1). Tiene una longitud aproximada de $170 \mathrm{~km}$, en sentido Norte-Sur y sus mayores alturas se ubican alrededor de los 2000 msnm.

Presenta características diferentes en ambas laderas. Hacia el oeste, la ladera es muy abrupta mientras que la ladera este presenta una pendiente suave que conforma tres pisos altitudinales: el primero con característica de bosque entre los 700 y los 1500 msnm, seguido de un piso de arbustos y pastos entre los 1500 y los 1800 msnm, mientras que en la cumbre, superando los 1800 msnm, la vegetación toma características de pastizal de altura. Éste último, se encuentra surcado por quebradas separadas 


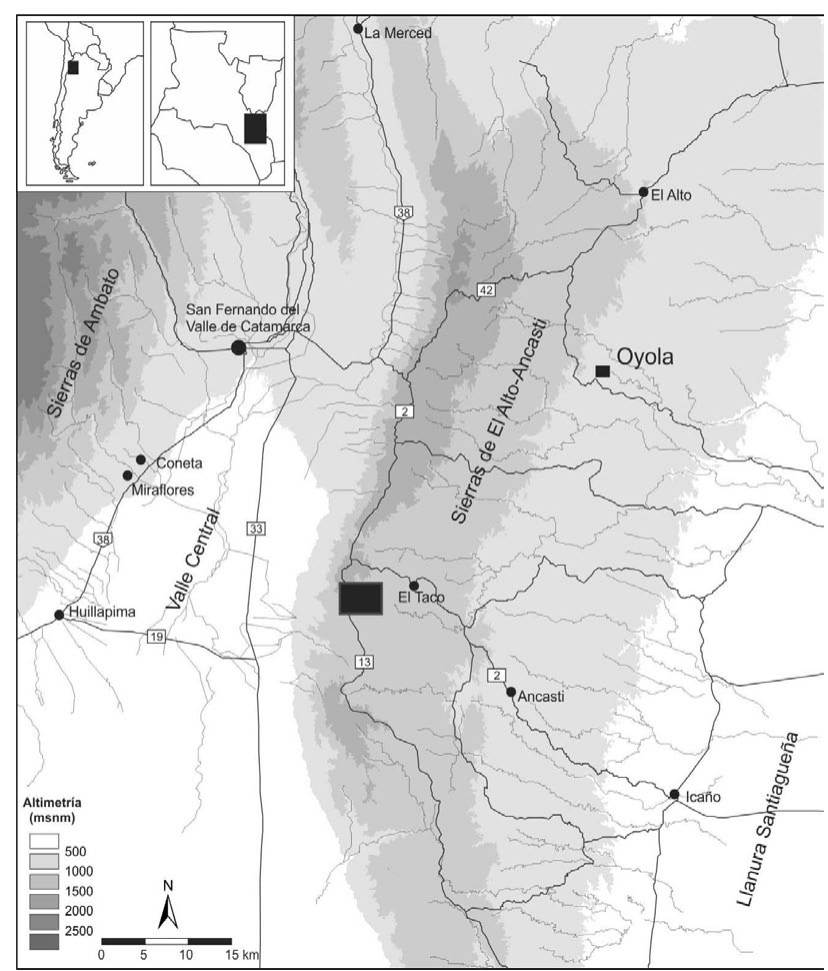

Figura 1: Localización de la sierra de El Alto-Ancasti

Figure 1: Location of El Alto-Ancasti mountains

por lomadas, lo que resulta en paisajes positivos y negativos. Las áreas elevadas presentan suelos rocosos, mientras que en las quebradas se depositan sedimentos aluviales.

En el marco de las investigaciones realizadas en la sierra se han excavado varios sitios, que permitieron conocer un poco más profundamente la historia de la ocupación de esta región (Duglosz y Nuñez Regueiro, 2005; Quesada, et al., 2012). Para el caso particular de este trabajo y de la tecnología lítica, nos hemos centrado en el estudio de dos sitios contemporáneos, pero con diferencias significativas. Hasta el momento contamos con seis fechados radiocarbónicos para ambos sitios que los ubican en la segunda mitad del primer milenio de la era.

Por un lado, Oyola 7 (OY7) (Figura 2) es una cueva con pinturas rupestres ubicada en la parte baja de la sierra, aproximadamente a $900 \mathrm{msnm}$. Su interior posee un tamaño aproximado de $14 \mathrm{~m}$ de largo, $4 \mathrm{~m}$ de ancho y
1,6 m de alto, por lo que es una de las cuevas pintadas más grandes de la localidad de Oyola. Posee dos accesos. Al oeste, la entrada presenta no más de $1 \mathrm{~m}$ de alto, y se abre a un gran playón de una gran roca granítica desde donde se puede apreciar el paisaje circundante. La boca este, es más amplia, pero con un acceso más restringido debido a la existencia de grandes rocas y abundante vegetación.

En el interior del abrigo se localizaron dos morteros y se observa un fuerte proceso de sedimentación. Se documentaron 77 motivos rupestres, confeccionados en colores blancos, negros y rojos (Gheco, 2017). En total en OY7 se recuperaron 809 hallazgos, principalmente material lítico (419 hallazgos). Le siguen en cantidad los fragmentos cerámicos (270 hallazgos) y los fragmentos óseos (117 hallazgos). También se hallaron 3 fragmentos de metal y varios fragmentos pequeños de cáscaras de huevo.

Pensamos que OY7 fue visitado y pintado en un largo discurrir histórico. En estas visitas, se ocupó la cueva, se encendieron fogones, posiblemente se cocieron alimentos y se realizaron actividades en las cuales participaron los instrumentos líticos.

El otro sitio analizado es El Taco 19 (ET19) (Figura 3). Se trata de un sitio de habitación a cielo abierto, ubicado en la cumbre de la sierra y vinculado a extensos sectores agrícolas aterrazados. La tecnología lítica junto con la arquitectura local permitió interpretar un uso del espacio de larga duración, con un compromiso con el territorio que implica un uso de recursos locales, la conservación de los suelos agrícolas y un modo de vida campesino (Quesada, et al., 2012).

E19 cuenta con 27 recintos asociados que presentan muros construidos mediante una doble hilera de lajas ubicadas de forma vertical clavadas en el sedimento y que pueden alcanzar una altura de 1 metro, completados por la colocación de bloques irregulares. El intersticio entre las lajas era completado con tierra, mientras que en el caso de los bloques irregulares se completaba con mortero.

La información obtenida de la excavación de 2 de estos 27 recintos, así como también del análisis de los materiales recuperados ha permitido interpretar al recinto

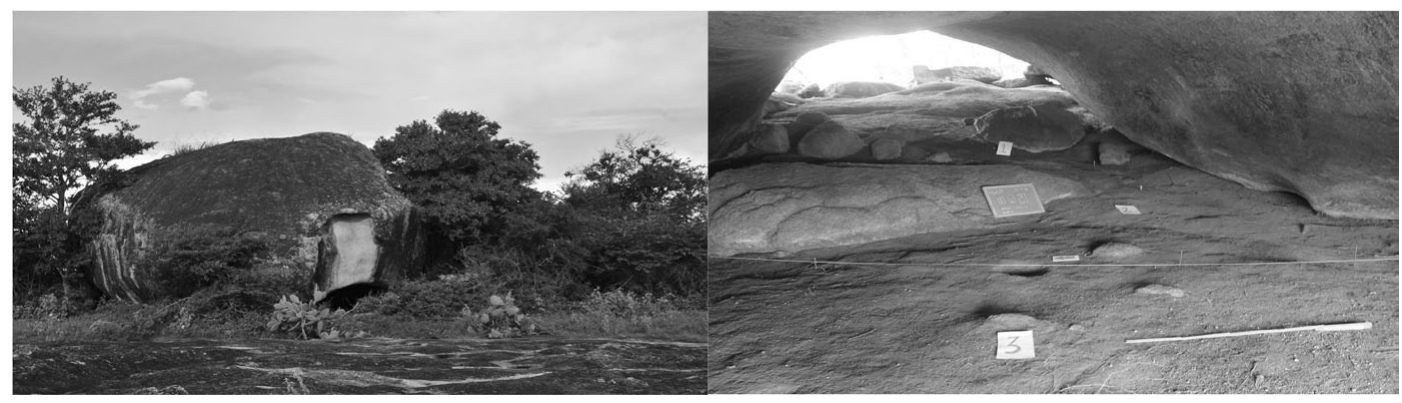

Figura 2: Vista frontal e interior de OY7

Figure 2: General and internal view of OY7 


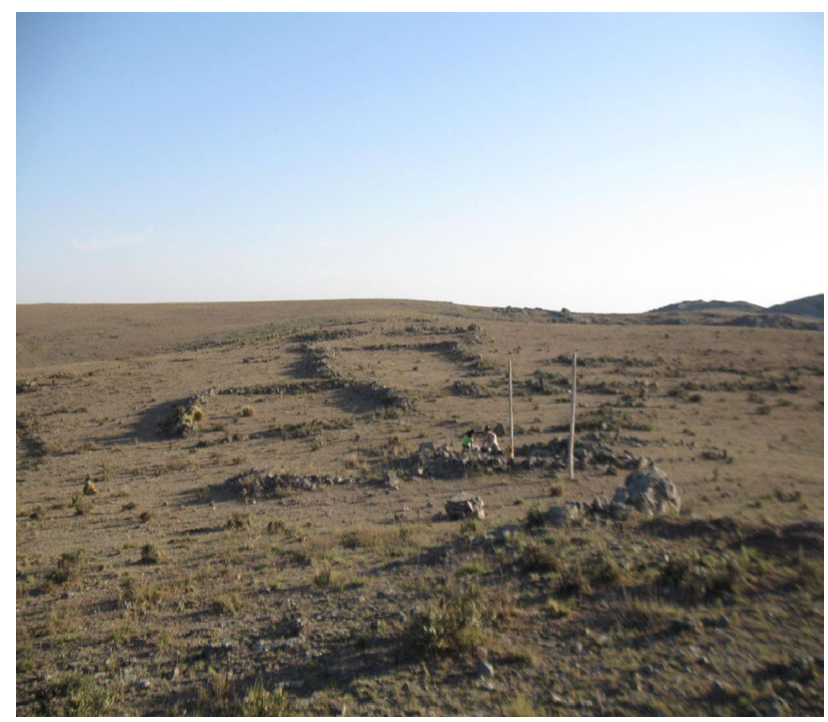

Figura 3: Vista general de ET19

Figure 3:General view of ET19

1 como un área de actividades, con un piso de ocupación cortado por pozos, algunos de ellos conteniendo vasijas cerámicas y recubiertas con lajas. Por su parte, el recinto 2 fue interpretado como una posible área de actividades constituida por acciones de vertido de residuos, donde hay presencia de cenizas, fragmentos óseos de gran tamaño, fragmentos cerámicos, desechos de talla, etc. Finalmente, el espacio exterior fue interpretado como la construcción de un gran pozo utilizado como vertedero y posteriormente tapado, posiblemente luego de la construcción del recinto 2 .

Es relevante remarcar la importancia del recurso Camelidae para los pobladores de estos dos sitios de la sierra, con indicadores del procesamiento, consumo y descarte de estos animales. También es importante el aprovechamiento de una variedad de especies silvestres (Ahumada y Moreno, 2015-2016). Este uso de una amplia diversidad de especies tendría un fuerte impacto en las estrategias de obtención de recursos, así como también consecuencias en la organización y reproducción de la vida cotidiana.

\section{Materiales líticos en la sierra}

En ET19, como ya se mencionó, la materia prima utilizada para la confección de instrumentos es casi exclusivamente el cuarzo. Cerca del sitio se detectaron al menos tres afloramientos de esta materia prima, a distancias que varían entre 300 y $420 \mathrm{~m}$ de distancia. En estas canteras se observan grandes bloques de cuarzo, y también desechos de talla y núcleos. Es relevante el hallazgo de percutores similares a los identificados en ET19 en estas canteras, lo que indicaría la realización de actividades de tallado y/o testeo en el lugar (Moreno y Sentinelli, 2014; Moreno, 2015).
El conjunto lítico recuperado $(n=728)$ cuenta con un alto porcentaje de desechos de talla, que abarca el $89 \%$ de la muestra $(n=646)$, le siguen en cantidad instrumentos manufacturados con el $8 \%(n=63)$, núcleos con el $2 \%$ $(n=14)$ y finalmente, percutores en un $1 \%(n=5)$. La cantidad de desechos de talla, así como el hallazgo de percutores y núcleos en el interior de los recintos evidencian la presencia de la totalidad de las etapas de manufactura lítica.

Los percutores identificados son todos de cuarzo, de forma elíptica y con presencia de picado y astillado. Se identificó en los percutores una superficie que se encontraba pulida y que en un principio fue interpretado como una posible reutilización de los mismos para tareas de raspado. Sin embargo, durante la experimentación, se observó que los percutores utilizados desarrollaban esta superficie como resultado del material que se iba separando del percutor como producto del picado (Moreno, 2015).

Se pudo observar en el conjunto algunos especímenes que presentan características que indican la utilización de talla bipolar. Estos alcanzan el 10\% del conjunto pero podría estar sub-representada por la dificultad para identificar este tipo de técnica. Particularmente nos referimos a la ausencia de talones, presencia de talones filiformes, estrías encontradas y plataformas de percusión machacadas o con varios puntos de percusión, resultado de los golpes realizados sobre el núcleo para la obtención de lascas. Además, se trata, en un mayor porcentaje, de desechos de modulo alargado y menor espesor que aquellos manufacturados mediante talla directa (Moreno y Sentinelli, 2014; Moreno, 2015).

Siendo la obtención de nódulos y núcleos de cuarzo una tarea relativamente simple dado que las canteras se encuentran a pocos metros de distancia, la utilización de esta técnica remitiría a la dureza de la materia prima. A través de la talla bipolar, se obtendrían formas bases aptas para la manufactura de instrumentos que difícilmente podían ser preparadas mediante la talla directa. Esta última genera desechos de gran espesor, lo que vuelve una tarea compleja la obtención de biseles aptos para la preparación de filos.

En OY7 la muestra de materiales líticos está compuesta por un total de 419 especímenes. El 74\% $(n=308)$ del conjunto corresponde a desechos de talla, conformando los instrumentos el 26\% ( $n=111)$. No se recuperaron percutores ni núcleos. Al igual que en ET 19, el cuarzo es prácticamente la única materia prima utilizada, concentrando el $96 \%$ del conjunto, mientras que la cuarcita y la filita representan el $4 \%$.

El cuarzo, se encuentra presente a pocos metros de la cueva, donde se ubican vetas de esta materia prima, con bloques de diferentes tamaños pero fácilmente 


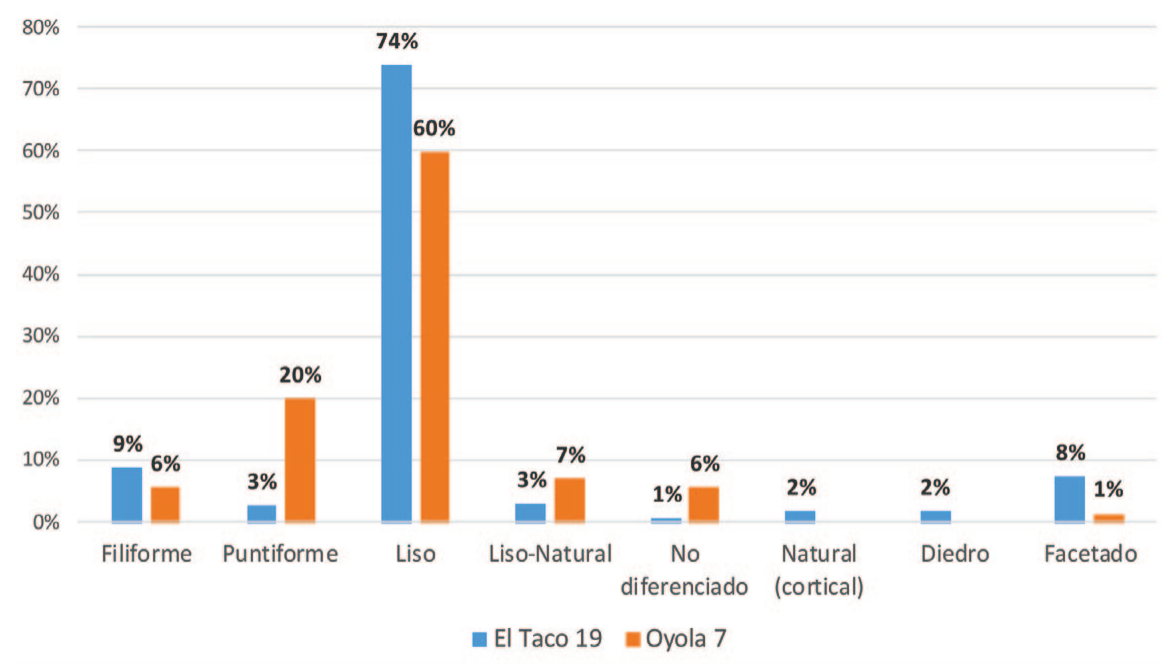

Figura 4: Tipos de talón presentes en los sitios OY7 y ET19

Figure 4: Types of striking platforms found at OY7 y ET19 transportables (Moreno y Egea, 2015; Egea, 2016). En cuanto a las técnicas de talla, solo registramos el uso de talla directa, debido a la mayor representatividad de talones lisos (60\%), aunque hay un porcentaje importante de talones puntiformes (20\%) que podrían estar relacionados al uso de la técnica bipolar, aspecto sobre el cual volveremos más adelante (Figura 4).

La ausencia de núcleos y percutores y las técnicas de talla marcan el ingreso a la cueva de formas base grandes, utilizadas para la preparación de diferentes tipos de instrumentos de manufactura simple. El conjunto presenta características esperables en un contexto en donde primaron las actividades de formatización y reactivación de filos, es decir, instancias avanzadas del proceso de producción lítica (Egea, 2016).

En resumen, los conjuntos de ambos sitios tienen como característica principal el estar manufacturados casi totalmente en cuarzo. Entendemos que esto implicaría la presencia de pobladores locales con altos conocimientos de su entorno y de los recursos disponibles.
Una particularidad llamativa del cuarzo utilizado es la identificación de diferentes calidades tecnológicas y estéticas sin que dicho factor afectara las elecciones de los talladores, variando desde fragmentos cristalinos hasta otros lechosos y otros que presentan vetas de diferentes colores, predominantemente rojo (Moreno y Sentinelli, 2014; Moreno, 2015; Egea, 2016). Prácticamente son inexistentes los desechos e instrumentos manufacturados en otras materias primas que implicarían el necesario traslado hacia otros paisajes para su obtención, pero que podrían otorgar una calidad para la talla mayor, así como mayor simplicidad para la manufactura de instrumentos. Las otras materias primas identificadas son cuarcita, filita y sílices.

La técnica de talla utilizada, en ambos conjuntos líticos, habría sido principalmente la talla directa, observándose en varios especímenes talones de gran tamaño, así como también un espesor importante de estos desechos en el caso de ET19. En OY7 los espesores son menores, pero esto tendría que ver con la ausencia en la cueva de las primeras etapas de manufactura, a diferencia de ET19

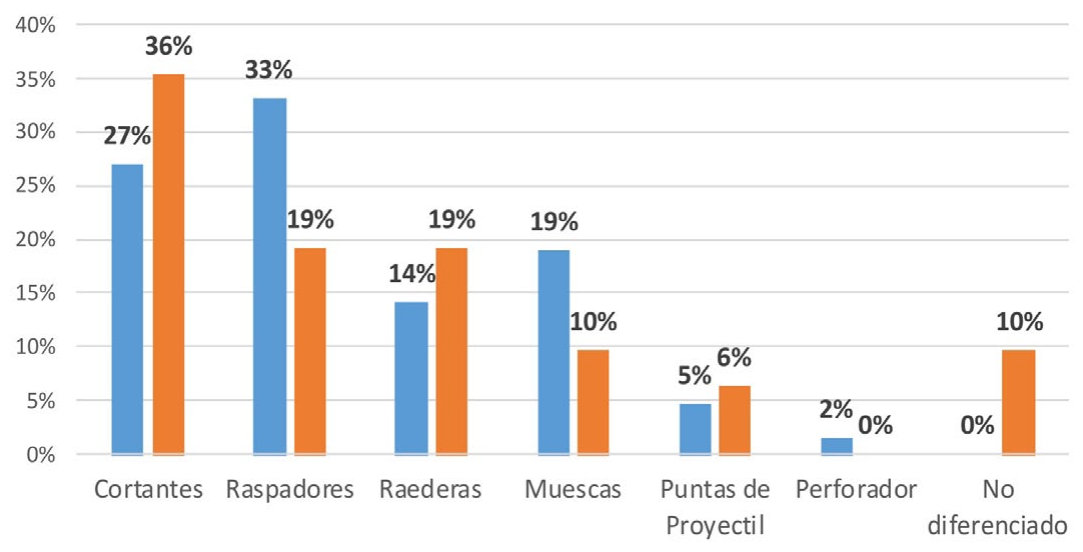

-El Taco 19 Oyola 7
Figura 5: Tipos de filos presentes en OY7 y ET19

Figure 5: Types of instruments identified at OY7 y ET19 


\begin{tabular}{|c|c|c|c|c|c|}
\hline \multirow[b]{2}{*}{ Variables } & \multirow[b]{2}{*}{ Estados de variables } & \multicolumn{2}{|c|}{ Talla Directa } & \multicolumn{2}{|c|}{ Talla Bipolar } \\
\hline & & $\mathrm{n}$ & $\%$ & $\mathrm{n}$ & $\%$ \\
\hline \multirow{4}{*}{$\begin{array}{c}\text { Estado de } \\
\text { los } \\
\text { desechos }\end{array}$} & Lasca entera & 59 & 56 & 12 & 17 \\
\hline & Lasca fracturada con talón & 33 & 31 & 29 & 43 \\
\hline & Lasca fracturada sin talón & 14 & 13 & 27 & 40 \\
\hline & Totales & 106 & 100 & 68 & 100 \\
\hline \multirow{7}{*}{$\begin{array}{l}\text { Tipo de } \\
\text { desecho }\end{array}$} & Lasca angular & 14 & 13 & 37 & 54 \\
\hline & Lasca de arista & 33 & 31 & 10 & 15 \\
\hline & Lasca no diferenciada & 5 & 7 & 4 & 6 \\
\hline & Lasca plana & 17 & 16 & 7 & 10 \\
\hline & Lasca primaria & 13 & 11 & 4 & 6 \\
\hline & Lasca secundaria & 24 & 22 & 6 & 9 \\
\hline & Totales & 106 & 100 & 68 & 100 \\
\hline \multirow{6}{*}{$\begin{array}{l}\text { Tipo de } \\
\text { Talón }\end{array}$} & Filiforme & 5 & 5 & 33 & 80 \\
\hline & Puntiforme & 10 & 11 & 8 & 20 \\
\hline & Liso & 55 & 60 & 0 & 0 \\
\hline & Liso-Natural & 19 & 21 & 0 & 0 \\
\hline & No diferenciado & 3 & 3 & 0 & 0 \\
\hline & Totales & 92 & 100 & 41 & 100 \\
\hline \multirow{7}{*}{$\begin{array}{l}\text { Tamaño } \\
\text { relativo }\end{array}$} & Muy grande & 3 & 3 & 0 & 0 \\
\hline & Grande & 20 & 19 & 0 & 0 \\
\hline & Mediano grande & 23 & 22 & 6 & 9 \\
\hline & Mediano pequeño & 48 & 45 & 19 & 28 \\
\hline & Pequeño & 12 & 11 & 36 & 53 \\
\hline & Muy pequeño & 0 & 0 & 7 & 10 \\
\hline & Totales & 106 & 100 & 68 & 100 \\
\hline \multirow{8}{*}{$\begin{array}{l}\text { Módulos } \\
\text { longitud- } \\
\text { anchura }\end{array}$} & Corto anchisimo & 3 & 3 & 7 & 10 \\
\hline & Corto muy ancho & 21 & 20 & 7 & 10 \\
\hline & Corto ancho & 26 & 24 & 12 & 18 \\
\hline & Mediano alargado & 5 & 5 & 11 & 16 \\
\hline & Mediano normal & 50 & 47 & 15 & 22 \\
\hline & Laminar angosto & 0 & 0 & 4 & 6 \\
\hline & Laminar normal & 1 & 1 & 12 & 18 \\
\hline & Totales & 106 & 100 & 68 & 100 \\
\hline
\end{tabular}

Tabla 1: Variables consideradas en el análisis de los conjuntos experimentales

Table 1: Variables considered in the analysis of the experimental assemblages donde encontramos percutores y núcleos que evidencian la realización de todas las etapas de producción. En el caso de ET19 además de talla directa hay presencia de talla bipolar.

Pensamos que los talladores buscaban formas base para la preparación de instrumentos, los que muestran una manufactura simple y una variabilidad de filos de rápida preparación y útiles para la realización de diferentes actividades (Moreno y Egea, 2015; Egea, 2016). Para su manufactura, la técnica se basó principalmente en la talla unifacial marginal o ultramarginal. Estos instrumentos habrían participado de una diversidad de necesidades cotidianas, siendo la obtención de materia prima simple y la manufactura expeditiva en pos de resolver problemas cotidianos (Figura 5).

\section{Experimentación}

El objetivo de este trabajo es presentar los primeros resultados del análisis efectuado sobre los materiales producto de una experimentación realizada por la Dra. Patricia Escola y donde participé junto al Dr. Enrique Moreno, cuya meta era evaluar las características mecánicas del cuarzo, así como también las posibles técnicas de talla utilizadas para la obtención de formas 
base. Para ello se utilizaron nódulos recolectados de una de las canteras cercanas a ET19, así como también percutores de cuarzo de diferentes tamaños.

Se tallaron tres nódulos de cuarzo para reducirlos mediante la implementación de talla directa y de talla bipolar. Concretamente, el interés se centra en evaluar las características específicas de los desechos obtenidos en cada una de estas actividades para luego poder compararlas con los conjuntos líticos arqueológicos recuperados en los sitios de estudio antes mencionados.

Para ello, se analizaron los conjuntos de desechos líticos experimentales a través de metodologías cuantitativas. Las variables tomadas en consideración fueron: estado y tipo de desecho, tipo y ancho del talón y tamaño y módulo de la pieza. Los resultados alcanzados nos permitieron inferir que existen diferencias y similitudes en los desechos producidos mediante las dos técnicas empleadas.

En primer lugar, la experimentación realizada mostró que la elección de los nódulos era un factor muy relevante ya que la presencia de microalteraciones internas genera la ruptura total del núcleo, haciendo casi imposible la obtención de formas base. Por lo tanto, la elección de los nódulos requería de dicho conocimiento basado, creemos, en el testeo en la cantera previó a su traslado al sitio para la obtención de formas base (Nami, 1992; Moreno, 2015).

La muestra experimental consta de 174 desechos. La frecuencia de especímenes fracturados, que alcanza un porcentaje del $60 \%$, podría ir de la mano de la dureza propia de esta materia prima, ya que tendería a fracturarse o astillarse fácilmente y a partir de la realización de un golpe se producen gran cantidad de desechos de tamaños variables y mucho polvillo (Moreno 2015). Los desechos de talla directa $(n=106)$ mostraron especímenes fracturados en un 44, 3\%, mientras que los de talla bipolar ( $n=68)$ llegan al $82 \%$.

Los desechos mostraron en el caso de la reducción de núcleos por percusión directa, mayoría de talones lisos (61\%) y lisos-naturales (21\%), con un tamaño promedio de 21,6 mm. En la reducción de núcleos por talla bipolar mostraron talones filiformes (80\%) y puntiformes (20\%) y algunas piezas con machacado en ambos extremos.

En cuanto a los tamaños representados destacan los medianos pequeños, medianos grandes y grandes en la talla directa, y pequeños y medianos pequeños en el caso de la talla bipolar. Por otro lado, mientras que los módulos son mayormente medianos normales (47\%), seguido de cortos anchos (24\%) y cortos muy anchos (20\%) en la talla directa, en la talla bipolar son variables, predominando levemente los cortos (38\%) y medianos (38\%) seguidos de los laminares (24\%). Finalmente, en cuanto a los espesores el promedio fue de 11,9 mm en talla directa y 6,1 $\mathrm{mm}$ en talla bipolar (Tabla 1).

Los resultados alcanzados permiten inferir que existen diferencias y similitudes en la utilización de técnicas de reducción lítica cuando nos enfrentamos a una materia prima con las características que presenta el cuarzo.

En los experimentos de talla bipolar se observó que los desechos presentan atributos típicos de este tipo de talla, tales como marcas de contragolpe distales, talones filiformes o puntiformes, aspectos que habíamos notado en el conjunto arqueológico de ET19. Asimismo, esto nos permite pensar para el caso de OY7, donde el porcentaje de talones puntiformes (20\%) es significativo, que podría deberse al uso de este tipo de percusión, si bien no registramos otros rasgos característicos. En cuanto a las marcas de bulbo u ondas de percusión no se registraron, ya que en general el cuarzo no suele presentarlas. No se detectaron tampoco, como sí en el conjunto arqueológico, mayoría de módulos alargados, terminación en forma de vírgula o la ausencia de talones. Sin embargo, esta puede ser una cuestión de dificultad en su identificación.

Al igual que en los conjuntos arqueológicos, en los experimentos se registraron altos índices de fragmentación. Esto tiene relación con el comportamiento físico-mecánico del cuarzo. En el caso de la talla bipolar, la mayor intensidad de las percusiones tiende a fracturar las lascas, generando así una mayor cantidad de desechos por experimento (Curtoni, 1996; Pautassi y Sario, 2014; Sario y Pautassi, 2015).

En cuanto al tamaño de los desechos se observa que la mayor parte de aquellos removidos mediante percusión directa son de mayor tamaño que los producidos por talla bipolar. Lo mismo ocurre con los espesores, aunque existe mayor variación. Esto es compatible, además, con lo visto en el conjunto arqueológico de ambos sitios.

\section{Comentarios finales}

Los resultados alcanzados nos muestran que el uso de percusión directa y bipolar estuvo presente en ET19. Cabe la posibilidad de que esta última técnica también en OY7, por el porcentaje significativo de talones puntiformes observado. El uso de diferentes técnicas de talla para la obtención de instrumentos muestra un alto grado de conocimiento de las reacciones de esta materia prima por parte de los talladores.

Algunas investigaciones realizadas en la provincia argentina de San Luis, avanzaron en cuanto a experimentaciones en esta materia prima, planteando la utilización tanto de talla bipolar como de talla directa en conjuntos manufacturados en cuarzo, obteniendo algunos resultados diferentes a los alcanzados aquí (Pautassi y Sario, 2014; Sario y Pautassi, 2015). Estos autores plantean que en los resultados 
experimentales se da una mayor variabilidad de los tamaños de los productos bipolares con respecto a los obtenidos por talla directa. En el caso de los desechos producidos por percusión directa, los tamaños de las lascas enteras son mayormente pequeños y medianos pequeños. En la talla bipolar son mayormente pequeños. Asimismo, se plantea que los desechos obtenidos mediante percusión directa poseen menores espesores que aquellos manufacturados mediante talla bipolar. Según los autores esto se debe a que en la talla bipolar se posee menos control en la remoción de lascas, por ello los desechos así removidos tienden a ser más espesos que los obtenidos mediante talla directa. Estas diferencias en los resultados obtenidos en las distintas experimentaciones, podrían tener que ver con los tipos o variedades de cuarzo y sus características específicas, y también con la aplicación de las técnicas de talla. Por esta razón es necesaria la realización de más experimentaciones, abarcando y problematizando estos aspectos.

Como se mencionó, estos experimentos fueron realizados con el fin de obtener una colección de referencia para poder comparar los diferentes atributos de los desechos de talla arqueológicos con los experimentales. A partir de esta primera aproximación se pudo constatar que las dos técnicas se hallaban presentes en los sitios de la sierra de El Alto-Ancasti. La experimentación sirvió de respaldo a la idea de que en el sitio ET19 se estaban desarrollando tanto las etapas iniciales de producción como las intermedias y finales mediante las dos técnicas de percusión. Por su parte, en la cueva OY7 se estaban realizando los momentos finales de la reducción de artefactos mediante talla directa, aunque cabe la posibilidad, por la cantidad de talones puntiformes registrados, de estar también frente a talla bipolar. Podría pensarse que en la cantera o en la cueva se habría utilizado la talla bipolar para la obtención de formas base y/o la preparación de instrumentos.

Nos resta profundizar la experimentación con esta materia prima, con la realización de mayores pruebas y también realizar actividades de corte, raspado, etc. sobre diferentes sustancias para evaluar la eficiencia y la durabilidad de estos filos. Podría pensarse que el cuarzo, por su muy alta dureza debería ser bueno para la manufactura de instrumentos, sin embargo, lo que se observa es que los biseles manufacturados se astillan fácilmente. Es por ello que habría que avanzar la experimentación en este sentido.

A su vez, sería útil experimentar con muestras de otros sectores de las sierras de El Alto-Ancasti, y zonas cercanas, estableciendo una buena clasificación petrológica y caracterización de las distintas variedades que permita la comparación y la suma de casos para el análisis de la tecnología lítica de cuarzo.

Catamarca, 25 de enero 2018

\section{Agradecimientos}

Quiero agradecer a Enrique Moreno y Patricia Escola por el apoyo y observaciones y especialmente por la experimentación realizada. A los integrantes del equipo del proyecto El Alto-Ancasti que participaron de las actividades de campo. Este trabajo se realizó en el marco de diversos proyectos financiados por la Secretaría de Ciencia y Técnica de la Universidad Nacional de Catamarca, la Agencia Nacional de Promoción Científica y Tecnológica y el Fondo Nacional de las Artes.

\section{Referencias citadas}

Ahumada, M., y Moreno, E. (2015). La escala domestica y los animales. Tratamiento diferencial de partes esqueletarias y distribucion diferencial intra-sitio en EL Taco 19 (El Alto-Ancasti, Catamarca). Anales de Arqueologia y Etnologia, 70-71, 105-117.

Ballin, T.B. (2008). Quartz technology in Scottish prehistory. Scottish Archaeological Internet Report, (SAIR), 11.

Baqueiro Vidal, S. (2006). La producción lítica del yacimiento neolítico de O Regueiriño (Moaña, Pontevedra). Cuadernos de Estudios Gallegos, 53 (119), 53-83.

Caminoa J. M. (2016). Un estudio de tecnología lítica desde la antropología de las técnicas: el caso del Alero Deodoro Roca ca. 2970 AP. Ongamira, Ischilín, Córdoba. South American Archaeology Series, 26. Oxford: Archaeopress.

Carrera Aizpitarte, M. (2017). Avances en los estudios arqueológicos desarrollados en la sierra de San Luis. EI sitio Club El Trapiche (departamento Coronel Pringles, provincia de San Luis). Anales de Arqueología y Etnología, $72(2), 191-217$.

Chaparro, M. G. (2005). La organización de la tecnología lítica en sociedades pastoriles prehistóricas (desde ca $2.000 \mathrm{AP}$ ) en la quebrada de Inca Cueva. El caso de la cueva 5 (Jujuy, Argentina). Arqueología, 11, 9-47.

Cousseran, S. (2002). Les inclusions fluides, un outil pour la discrimination des quartz archéologiques. Application au problème de circulation du quartz dans les Alpes occidentales et lombardes. Réalisation d'un premier référentiel de données sur les quartz alpins. Bulletin de la Société préhistorique française, 99 (4), 833-838.

Curtoni, R. (1996). Experimentando con bipolares: indicadores e implicaciones arqueológicas. Relaciones de la Sociedad Argentina de Antropología, XXI, 187-214.

Dlugosz, J. C. y Núñez Reguero, V. (2005). Arqueología del Departamento El Alto: relaciones entre sociedades 
del pasado. Actas de VII Jornadas Regionales en Humanidades y Ciencias Sociales. UNJu.

Driscoll, K. (2010). Understanding quartz technology in early prehistoric Ireland, vol.1. Dublin: MS. University college

Egea, D. (2016). Practicas tecnológicas líticas entre las sociedades del este catamarqueño durante la segunda mitad del Primer milenio D.C. La Zaranda de Ideas, 14 (1), 55-70.

Elías, A. (2012). Tecnología lítica en el período tardío (ca. 1100-550 años AP) de Antofagasta de la Sierra (Provincia de Catamarca, Puna Meridional Argentina. Relaciones de la Sociedad Argentina de Antropología, XXXVII (1), 19-41.

Escola, P. (2004). Variabilidad en la explotación y distribución de obsidianas en la Puna meridional argentina. Estudios Atacameños, 28,9-24.

Fabregas Valcarce, R. y Rodríguez Rellan, C. (2008). Gestión del cuarzo y la pizarra en el Calcolítico Peninsular: El "Santuario" de El Pedroso (Trabazos de Aliste, Zamora). Trabajos de Prehistoria, 65 (1), 125-142.

Gaál, E. (2011). Un acercamiento preliminar al estudio de la base local de recursos líticos y al análisis artefactual en el poblado arqueológico de Rincón Chico (período Tardío), valle de Yocavil, Catamarca. Comechingonia, V (1), 1-38.

Gheco, L. (2017). Una arqueología de las prácticas vinculadas al arte rupestre. Arte, ritual y memoria en la Sierra de Ancasti (Catamarca) (Tesis doctoral). Facultad de filosofía y humanidades. Universidad Nacional de Córdoba, Córdoba.

González, A. R. (1998). Cultura La Aguada. Arqueología y diseños. Buenos Aires: Filmediciones Valero.

Gordillo, I., V. Zuccarelli, V., y Eguia, L. (2017). Las casas del sol naciente. Arqueología de la vertiente oriental de El Alto-Ancasti. En: B. N. Ventura, G. Ortiz y M. B. Cremonte (eds.), Arqueología de la vertiente oriental surandina. Interacción macro-regional, materialidades, economía y ritualidad (111-134). Ciudad Autónoma de Buenos Aires: Sociedad Argentina de Antropología.

Knutsson, H., Knutsson, K., Taipale, N., Tallavaara, M., y Darmaek, K. (2015). How shattered flakes were used: micro-wear analysis of quartz flake fragments. Journal of Archaeological Science: Reports, 2, 517-531.

Llamazares, A. (1999-2000). El arte rupestre de la cueva La Candelaria, provincia de Catamarca, Argentina. Publicaciones del CIFFYH, 50, 1-26.
Lombera Hermida, A. (2008). Quartz Morphostructural Groups and their Mechanical Implications. Annali dell'Universita degli Studi di Ferrara. Museologia Scientifica e Naturalistica Special, 101-105.

Manninen, M. A. (2016). The effect of raw material properties on flake and flake-tool dimensions: A comparison between quartz and chert. Quaternary International, 424, 24-31. Recuperado de http://dx.doi. org/10.1016/j.quaint.2015.12.096

Moreno, E. (2015). Materias primas, instrumentos líticos y prácticas domésticas en las serranías de El Alto-Ancasti, Catamarca. Cuadernos INAPL-Series Especiales, 2 (2), 141-160.

Moreno, E. y Egea, D. (2015). Visitas en el tiempo. Tecnología lítica de una cueva con arte rupestre en el este catamarqueño. Arqueología, 22, 223-232.

Moreno, E. y Sentinelli, N. (2014). Tecnología lítica en la sierra de El Alto-Ancasti, Catamarca. Cuadernos (UNJU), 45, 95-115.

Mourre, V. (1996). Les industries en quartz au Paléolithique. Terminologie, Methodologie et Technologie. Paleo, 8, 205-223.

Nami, H. (1992). El subsistema tecnológico de la confección de instrumentos líticos y la explotación de los recursos del ambiente: una nueva vía de aproximación. Shincal, 2, 33-53.

Nazar, C., Gheco, L. y De La Fuente, G. (2013). Las pinturas rupestres tardías de la cuenca de Ipizca-Icaño, Sierra de Ancasti, Catamarca, Argentina. Trabajo presentado en I Taller de Arqueología de la Sierra de Ancasti y zonas aledañas, Catamarca.

Pautassi, E. (2015). La talla y uso del cuarzo, una aproximación metodológica para la comprensión de contextos de cazadores-recolectores de Córdoba (Tesis doctoral). Facultad de Filosofía y Humanidades de la Universidad Nacional de Córdoba, Cordoba.

Pautassi, E. y Sario, G. (2014). La talla de reducción: aproximaciones experimentales para el estudio del cuarzo. Arqueoweb, 15, 3-17. http://pendientedemigracion.ucm. es/info/arqueoweb/pdf/15/Pautassi-Sario.pdf

Prous, A. P. (2004). Apuntes para análisis de industrias líticas. ORTEGALIA, Monografias de Arqueoloxía, Historia y Patrimonio 2. Ortigueira: Fundación Federico Maciñeira.

Quesada, M., Gastaldi, M. y Granizo, G. (2012). Construcción de Periferias y Producción de lo Local en las Cumbres de El Alto-Ancasti. Relaciones de la Sociedad 
Argentina de Antropología, 37, 435- 456.

Reinoso, D. (2017). Tecnología lítica del sitio Barranca I (Córdoba, Argentina): avances en el registro de las fuentes inmediatas de cuarzo. Revista Sociedades de Paisajes Áridos y Semi-Áridos, X, 195-220.

Robledo A., Cattáneo, R., y Conte, B. (2017). Tecnología lítica y uso del espacio en el alero Parque Natural Ongamira 1 (depto Ischilín, Córdoba, Argentina). Anales de Arqueología y Etnología, 72 (2), 219-244.

Rodríguez-Rellan, C. (2016). Variability of the rebound hardness as a proxy for detecting the levels of continuity and isotropy in archaeological quartz. Quaternary International, 424, 191-211. http://dx.doi.org/10.1016/j. quaint.2015.12.085
Sánchez Yustos P., Diez Martín, F., Domínguez-Rodrigo, M., y Tarriño Vinagre, A. (2012). Discriminación experimental de los rasgos técnicos en la talla bipolar y a mano alzada en lascas a través de los cuarzos de Naibor Soit (Garganta de Olduvai, Tanzania). Munibe (Antropologia-Arkeologia), 63, 5-26.

Sario, G. y Pautassi, E. (2015). Canteras-taller de cuarzo y un análisis de los conjuntos artefactuales del sitio Piedra Blanca (Copacabana, Córdoba). Revista Arqueología, 21 (2), 165-175.

Spott, E. (2005). Analysis of quartz in northern Wisconsin: Deficiencies, misconceptions and goals. Nebraska Anthropologist, 10, 115-128. 